\title{
Structural basis for specific recognition of Lys 63-linked polyubiquitin chains by tandem UIMs of RAP80
}

\author{
Yusuke Sato ${ }^{1,2}$, Azusa Yoshikawa ${ }^{1,2}$, \\ Hisatoshi Mimura ${ }^{1}$, Masami Yamashita ${ }^{1,3}$, \\ Atsushi Yamagata ${ }^{1}$ and Shuya Fukai ${ }^{1,3, *}$ \\ ${ }^{1}$ Structural Biology Laboratory, Life Science Division, Synchrotron \\ Radiation Research Organization and Institute of Molecular and Cellular \\ Biosciences, The University of Tokyo, Tokyo, Japan, ${ }^{2}$ Department of \\ Biological Information, Tokyo Institute of Technology, Yokohama, Japan \\ and ${ }^{3}$ Department of Medical Genome Sciences, Graduate School of \\ Frontier Sciences, the University of Tokyo, Chiba, Japan
}

RAP80 has a key role in the recruitment of the AbraxasBRCC36-BRCA1-BARD1 complex to DNA-damage foci for DNA repair through specific recognition of Lys 63-linked polyubiquitinated proteins by its tandem ubiquitin-interacting motifs (UIMs). Here, we report the crystal structure of the RAP80 tandem UIMs (RAP80-UIM1-UIM2) in complex with Lys 63 -linked di-ubiquitin at $2.2 \AA$ resolution. The two UIMs, UIM1 and UIM2, and the $\alpha$-helical interUIM region together form a continuous $60 \AA$ A-long $\alpha$-helix. UIM1 and UIM2 bind to the proximal and distal ubiquitin moieties, respectively. Both UIM1 and UIM2 of RAP80 recognize an Ile 44-centered hydrophobic patch on ubiquitin but neither UIM interacts with the Lys 63-linked isopeptide bond. Our structure suggests that the inter-UIM region forms a 12 A-long $\alpha$-helix that ensures that the UIMs are arranged to enable specific binding of Lys 63-linked diubiquitin. This was confirmed by pull-down analyses using RAP80-UIM1-UIM2 mutants of various length inter-UIM regions. Further, we show that the Epsin1 tandem UIM, which has an inter-UIM region similar to that of RAP80-UIM1-UIM2, also selectively binds Lys 63-linked di-ubiquitin.

The EMBO Journal (2009) 28, 2461-2468. doi:10.1038/

emboj.2009.160; Published online 18 June 2009

Subject Categories: structural biology

Keywords: crystallography; DNA repair; ubiquitin; ubiquitininteracting motif

\section{Introduction}

Ubiquitin is a highly conserved 76-residue protein that can be covalently attached to substrate proteins to regulate a wide variety of biological processes (Hicke, 2001; Glickman and Ciechanover, 2002; Miranda and Sorkin, 2007; Hofmann,

\footnotetext{
*Corresponding author. Institute of Molecular and Cellular Biosciences, The University of Tokyo, General Research Bldg 211, 1-1-1 Yayoi, Bunkyo-ku, Tokyo 113-0032, Japan. Tel.: + 8135841 7807;

Fax: + 8135841 7807; E-mail: fukai@iam.u-tokyo.ac.jp
}

Received: 20 April 2009; accepted: 20 May 2009; published online: 18 June 2009
2009; Wickliffe et al, 2009). The terminal carboxylate of ubiquitin is typically attached to the $\varepsilon$-amino group of substrate lysine residues. Ubiquitin itself has seven lysine residues and one terminal amino group, all of which can be attached to additional ubiquitin molecules, producing eight types of polyubiquitin chains (Kirisako et al, 2006; Wickliffe et al, 2009). Among these, Lys 48-linked polyubiquitin chains are the most abundant in vivo, and constitute the canonical signal for the degradation by the proteasome (Glickman and Ciechanover, 2002). Although the roles for other types of polyubiquitin chains are not fully understood, recent proteomic studies suggest that the Lys 11-linked chains may be abundant in vivo and that polyubiquitin chains linked by residues other than Lys 63 may be able to act as degradation signals for the proteasome and have partially redundant functions (Meierhofer et al, 2008; Xu et al, 2009). However, Lys 63-linked polyubiquitin chains seem to function primarily in contexts outside the targeting substrates of the proteasome and to play crucial roles in intracellular events, such as inflammatory signalling, endocytosis, vesicular trafficking, and DNA repair (Miranda and Sorkin, 2007; Verstrepen et al, 2008; Hofmann, 2009; Wickliffe et al, 2009). For instance, Lys 63-linked polyubiquitin chains signal sites of DNA damage in the course of DNA damage response (DDR) induced by DNA double-strand break (DSB) (Kinner et al, 2008; Yan and Jetten, 2008; Doil et al, 2009; Panier and Durocher, 2009; Stewart et al, 2009) (see Supplementary Figure S1). In this context, Lys 63-linked chains function as a signal to recruit a DDR protein complex containing BRCA1 (breast cancer susceptibility gene 1), BARD1 (BRCA1 associated RING domain 1), BRCC36 (BRCA1/2 containing complex subunit 36, also known as BRCC3), Abraxas (also known as CCDC98), and RAP80 (receptor-associated protein 80, also known as UIMC1), which has a critical function in DNA repair, cell-cycle checkpoint control, and maintenance of genome stability, particularly in association with breast and ovarian cancers (Kim et al, 2007; Sobhian et al, 2007; Wang and Elledge, 2007; Wang et al, 2007; Yan et al, 2007). BRCA1 and BARD1 form a complex to function as an E3 ubiquitin ligase (Brzovic et al, 2003; Christensen et al, 2007), whereas BRCC36 is a $\mathrm{Zn}^{2+}$-dependent de-ubiquitinating enzyme (DUB) (Sobhian et al, 2007; Shao et al, 2009). Abraxas serves as a scaffold to organize BRCA1-BARD1, BRCC36, and RAP80 (Wang and Elledge, 2007).

RAP80 acts as a linker responsible for recruiting the DDR complex to DSB sites through an Abraxas-interacting region and a ubiquitin-interacting motif (UIM) domain (Figure 1A and B) (Kim et al, 2007; Sobhian et al, 2007; Wang and Elledge, 2007; Wang et al, 2007; Yan et al, 2007; Yan and Jetten, 2008). The UIM domain of RAP80 contains a tandem double UIM, which recognizes Lys 63-linked polyubiquitin chains. This recognition is essential for the DNA-damageinduced recruitment of RAP80.

The UIM was initially identified in the ubiquitin-binding domains of the Rpn10 subunit of the 26 S proteasome (Young 

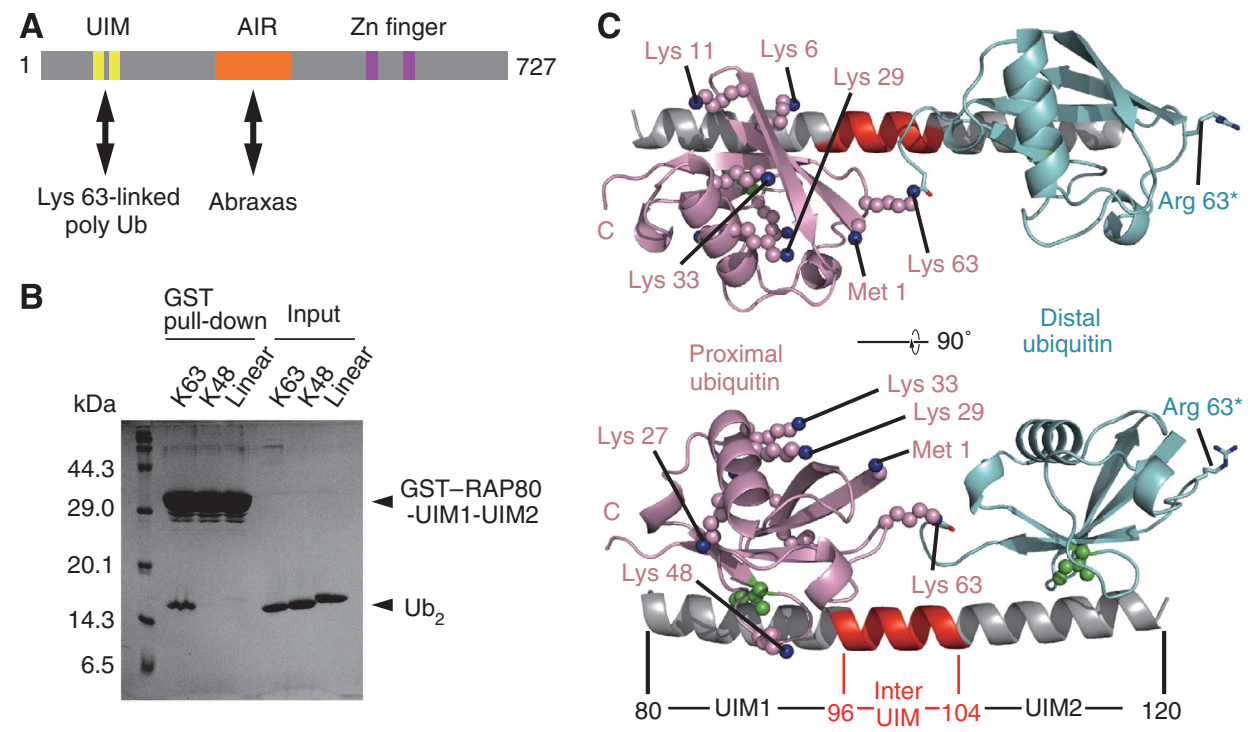

Figure 1 Overall structure of the RAP80-UIM1-UIM2-K63-Ub 2 complex. (A) Schematic view of mouse RAP80. UIM domain and AIR (Abraxasinteracting region) interact with Lys 63-linked polyubiquitin chains and Abraxas, respectively. (B) Pull-down assays using GST-fused RAP80UIM1-UIM2 to bind to K63-, K48- or linear $\mathrm{Ub}_{2}$. (C) The UIMs and inter-UIM region of RAP80-UIM1-UIM2 are coloured grey and red, respectively. The proximal and distal ubiquitin moieties are coloured pink and cyan, respectively. Ile 44 in the both of the ubiquitin moieties is shown as green spheres. Lysine residues and the N-terminal methionine of the proximal ubiquitin are shown as spheres. The K63R mutation in the distal ubiquitin (used to generate $\mathrm{K} 63-\mathrm{Ub}_{2}$ ) is shown by a stick model.

et al, 1998). The motif comprises a highly conserved sequence, $\phi$-X-X-Ala-X-X-X-Ser-X-X-e, where $\phi, e$, and $\mathrm{x}$ represent hydrophobic, acidic, and any amino-acid residue, respectively (Hofmann and Falquet, 2001). The UIM consists of a single $\alpha$-helix, and binds to mono-ubiquitin with a $K_{\mathrm{d}}$ value of 0.1-2 mM (reviewed in Hurley et al, 2006). The UIM structures have been reported in isolation or in complex with ubiquitin, and the interaction of UIMs with mono-ubiquitin has been well studied (Fisher et al, 2003; Swanson et al, 2003; Wang et al, 2005; Hirano et al, 2006). Given that the affinity between UIMs and mono-ubiquitin is low in general (Hurley et al, 2006), the observed robust interaction between RAP80 and Lys 63-linked polyubiquitin substrates is probably achieved by co-operative binding between multiple UIMs and ubiquitin chains interacting through an avidity-based mechanism, where binding by the first of multiple UIMs to one ubiquitin would position the second UIM favourably for interaction with a nearby ubiquitin in the chain. A recent study shows the Lys 63-linkage-specific avidity-based ubiquitin binding of RAP80 UIMs using domain swapping experiments (Sims and Cohen, 2009). To directly prove this mechanism, we have determined the structure of the tandem UIMs of RAP80 specifically bound to Lys 63-linked polyubiquitin chains and carried out structure-based mutagenesis. Here we report the crystal structure of the tandem UIMs of RAP80 in complex with a Lys 63-linked di-ubiquitin $\left(\mathrm{K} 63-\mathrm{Ub}_{2}\right)$ at a $2.2 \AA$ resolution. The structure of the complex and our structure-based mutagenesis show the structural basis for selective binding of Lys 63-linked polyubiquitin chains by RAP80.

\section{Results and discussion}

\section{Overall structure of a complex of RAP80-UIM1-UIM2 and K63-Ub}

We prepared two human RAP80 constructs (of residues 80-121 and residues 81-120) and five mouse RAP80 constructs (of residues 1-233, 65-125, 80-125, 80-121, $80-120$ ), all of which contain the RAP80 UIMs. Although all of these truncations formed a stable complex with $\mathrm{K} 63-\mathrm{Ub}_{2}$, only mouse RAP80 (residues 80-120) was successfully cocrystallized with the $\mathrm{K} 63-\mathrm{Ub}_{2}$. This region is referred to as RAP80-UIM1-UIM2 in this study. GST-fused RAP80-UIM1UIM2 binds $\mathrm{K} 63-\mathrm{Ub}_{2}$, but not Lys 48-linked or linear diubiquitin $\left(\mathrm{K} 48-\mathrm{Ub}_{2}\right.$ or linear $\left.\mathrm{Ub}_{2}\right)$ (Figure $\left.1 \mathrm{~B}\right)$. The crystal structure of the RAP80-UIM1-UIM2-K63-Ub 2 complex was solved by molecular replacement using the structure of a 1:1 stoichiometric complex of Hrs-UIM and ubiquitin (modified from PDB code: 2D3G) as a search model (Hirano et al, 2006). The atomic model was refined with an $R_{\text {free }}$ value of 0.268 at 2.2 A resolution.

As shown in Figure 1C, RAP80-UIM1-UIM2 forms a stoichiometric complex with $\mathrm{K} 63-\mathrm{Ub}_{2}$. UIM1 (residues 80-95) and UIM2 (residues 105-120) are connected by an $\alpha$-helical inter-UIM region and thus RAP80-UIM1-UIM2 forms a continuous $60 \AA$-long $\alpha$-helix. UIM1 and UIM2 interact with the proximal and distal ubiquitin moieties, respectively. The two RAP80-UIM1-UIM2-K63-Ub 2 complexes in the crystallographic asymmetric unit are very similar to one another overall. However, the $\mathrm{C}$-terminal tail conformation of the distal ubiquitin moieties differs among them (probably due to intrinsic flexibility), and the recognition of the proximal ubiquitin moiety is slightly different, with one complex affected by crystal packing. Hereafter, we will discuss the complex that is unaffected by crystal packing.

\section{Ubiquitin binding interfaces of RAP80-UIM1-UIM2}

Similar to several other well-characterized UIMs (Swanson et al, 2003; Wang et al, 2005; Hirano et al, 2006), both UIM1 and UIM2 of RAP80-UIM1-UIM2 recognize the Ile 44-centered hydrophobic patch of ubiquitin that is formed by the side chains of Leu 8, Ile 44, and Val 70 and aliphatic portions of 
the side chains of Arg 42, His 68, and Gln 49. The side chains of Phe 85, Leu 87, Ala 88, Leu 89, and Met 91, and aliphatic portions of the Gln 84 side chain in UIM1 form a hydrophobic surface to interact with the Ile 44-centered hydrophobic patch of the proximal ubiquitin (Figure 2A). Further, the $\mathrm{O} \varepsilon$ atom of Glu 81 and the $\mathrm{O} \gamma$ atom of Ser 92 in UIM1 hydrogen bond with the amide nitrogen atoms of Leu 73 and Gly 47 in the proximal ubiquitin, respectively. Similar to UIM1, the side chains of Leu 109, Leu 110, Ala 113, and Ile 114 and aliphatic portions of the side chains of Lys 112 and Glu 116 in UIM2 form a hydrophobic surface to interact with the Ile 44-centered hydrophobic patch of the distal ubiquitin (Figure 2B). The $\mathrm{O} \varepsilon$ atom of Glu 106 and the $\mathrm{O} \gamma$ atom of Ser 117 in UIM2 hydrogen bond with the amide nitrogen atoms of Leu 73 and Gly 47 in the distal ubiquitin, respectively. In addition, the side chain of Leu 118 and aliphatic portions of the Lys 105 side chain in UIM2 interact with Gly 47 and Leu 73 in the distal ubiquitin, respectively.
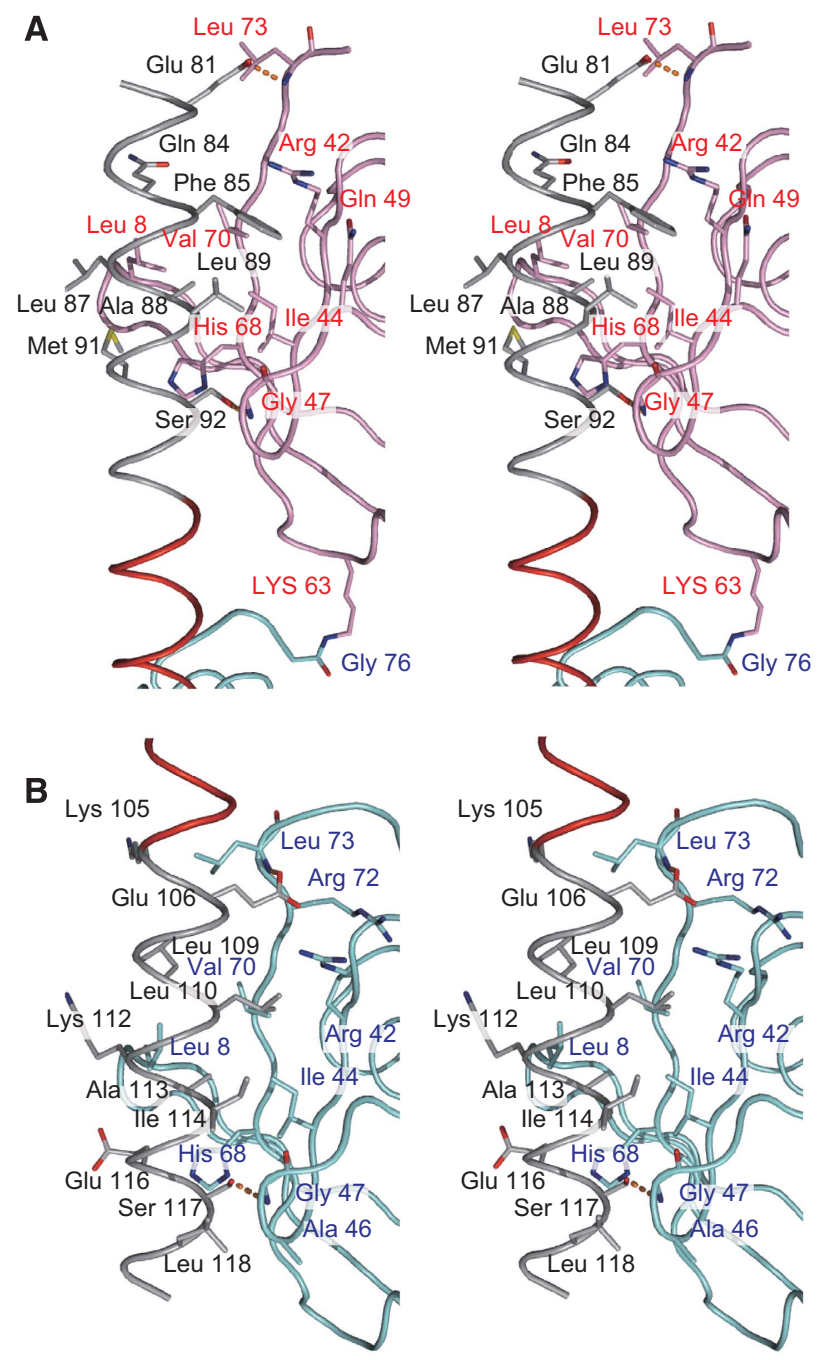

Figure 2 Stereo view of the interface between the ubiquitin moieties and RAP80-UIM1-UIM2. The colouring scheme is the same as in Figure 1. The labels of RAP80, the proximal, and distal ubiquitin moieties are coloured black, red, and blue, respectively. (A) The interface between the proximal ubiquitin and the UIM1. (B) The interface between the distal ubiquitin and the UIM2.

\section{Linkage selectivity of RAP80-UIM1-UIM2}

The structural details of ubiquitin binding by RAP80 UIM1 and UIM2 are similar to each other and those of other typical single UIM (Swanson et al, 2003; Wang et al, 2005; Hirano et al, 2006). In addition, neither UIM1 nor UIM2 directly interacts with the Lys 63-linked isopeptide bond of $\mathrm{K}_{63}-\mathrm{Ub}_{2}$ (Figures $1 \mathrm{C}$ and 2). Therefore, the order of UIM1 and UIM2 seems not to be critical for the selective recognition of Lys 63 isopeptide chains. However, the aminoacid sequences of the RAP80 UIM1 and UIM2 are highly conserved and differ from one another (Figure $3 \mathrm{~A}$ and B), implying that UIM1 and UIM2 might have distinct roles in the context of the specific recognition of $\mathrm{K} 63-\mathrm{Ub}_{2}$. To assess this possibility, we made the three possible artificial combinations of UIM1 and UIM2 (termed UIM1-UIM1, UIM2-UIM2, and UIM2-UIM1, respectively) and tested their ability to bind $\mathrm{K} 63-\mathrm{Ub}_{2}$ in GST pull-down assays (Figure 3C). As shown in Figure 3D, all three mutants retained the ability to bind $\mathrm{K} 63-\mathrm{Ub}_{2}$ with an apparent small decrease in affinity relative to wild type. Thus, the order of the UIM1 and UIM2 of RAP80-UIM1-UIM2 is not critical for the $\mathrm{K} 63-\mathrm{Ub}_{2}$ binding but is probably optimized to maximize the binding affinity. Note that bands of $\mathrm{K} 63-\mathrm{Ub}_{2}$ bound to the UIM1-UIM1 and UIM2-UIM2 were similarly stained by CBB, indicating that UIM1 and UIM2 have similar affinities for ubiquitin, consistent with the structural basis of ubiquitin recognition being similar for UIM1 and UIM2. The $\mathrm{C} \alpha$ atoms of the UIM1-proximal ubiquitin complex can be superposed onto those of the UIM2-distal ubiquitin complex with an r.m.s.d. value of $0.653 \AA$.

These results suggested that the inter-UIM region, rather than the details of the UIM1 and UIM2 interactions with ubiquitin, may have an important function in Lys 63-linkagespecific ubiquitin-chain recognition. Consistent with this idea, the inter-UIM lengths in all RAP80 homologues are perfectly conserved (See Figure 3A for examples). To test this hypothesis, we designed RAP80-UIM1-UIM2 mutants with inter-UIM regions of various lengths $(-1,+2$, and +4 ) (Figure 4A) and tested their abilities to bind to K63$\mathrm{Ub}_{2}, \mathrm{~K} 48-\mathrm{Ub}_{2}$, and linear $\mathrm{Ub}_{2}$ by GST pull-down assay. On the basis of our RAP80-UIM1-UIM2-K63-Ub 2 complex structure, the ubiquitin-binding surfaces of the UIM1 and UIM2 in the -1 and +2 mutants should be oriented on opposite sides of the $\alpha$-helix from one another (Figure 4B and Supplementary Figure S2). However, the distance between UIM1 and UIM2 in the +4 mutant is predicted to be $6 \AA$-longer than in wild-type RAP80-UIM1-UIM2, although the ubiquitin-binding surfaces of UIM1 and UIM2 should be oriented in nearly identical directions (Figure 4B and Supplementary Figure S2). As expected from our structure, none of the mutants bind K63-, K48-, or linear $\mathrm{Ub}_{2}$ (Figure $4 \mathrm{C}$ ), consistent with the appropriate length inter-UIM region being essential for specific recognition of $\mathrm{K} 63-\mathrm{Ub}_{2}$. As the inter-UIM region does not directly interact with the Lys 63-linked isopeptide bond, the inter-UIM region functions as a 'molecular ruler' to measure the inter-molecular distance between the proximal and distal ubiquitin moieties of $\mathrm{K} 63-\mathrm{Ub}_{2}$. In this context, UIM1 and UIM2 are located on opposite sides of the molecular ruler, and by virtue of being an $\alpha$-helix, the orientations of the surfaces available to bind to proximal and distal ubiquitin moieties are fixed. This significant role of the inter-UIM region in the specific binding with $\mathrm{K} 63-\mathrm{Ub}_{2}$ makes it highly 

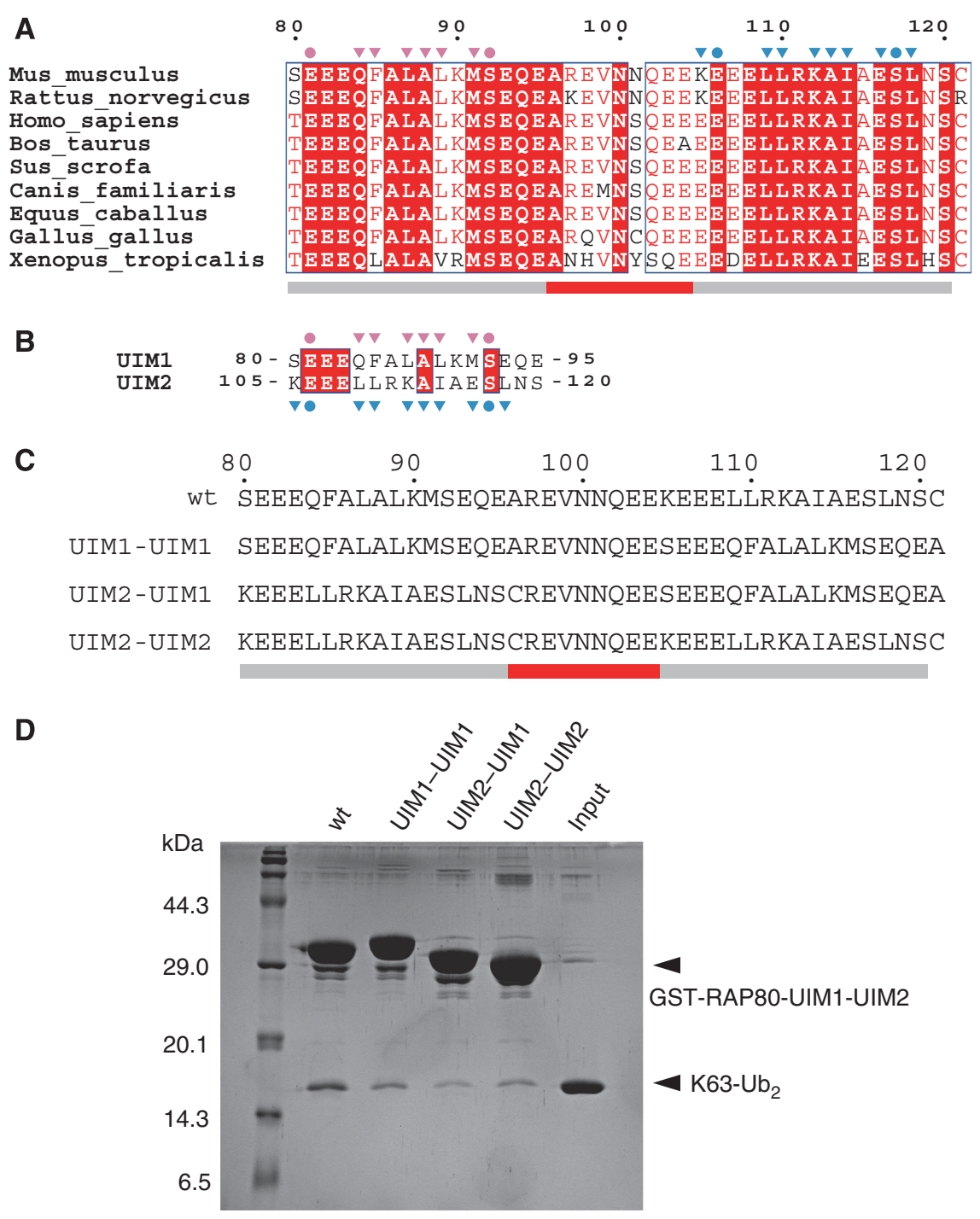

Figure 3 The order of the two UIMs of RAP80-UIM1-UIM2 is not critical for selective binding of K63-Ub ${ }_{2}$. (A) Amino-acid sequence alignment of the tandem UIMs of RAP80. The residue numbers of mouse RAP80 are indicated above the sequence alignment. Identical and more than $70 \%$ identical residues are highlighted by red background and red characters, respectively. Residues that hydrogen bond to the proximal and distal ubiquitin moieties are marked with pink and cyan circles, respectively. Residues involved in hydrophobic interactions with the proximal and distal ubiquitin moieties are marked with pink and cyan triangles, respectively. Grey and red bars below the alignment correspond to the UIMs and inter-UIM region, respectively. (B) Amino-acid sequence alignment of the UIM1 and UIM2 of the mouse RAP80. The symbols and colouring are the same as in Figure 3A. (C) Amino-acid sequences of the UIM1-UIM1, UIM2-UIM2, and UIM2-UIM1 of RAP80. (D) Pull-down assays using the GST-fused RAP80-UIM1-UIM2 mutants and $\mathrm{K} 63-\mathrm{Ub}_{2}$.

unlikely that UIM1 and UIM2 independently bind to distinct ubiquitin chains.

Among all the lysine residues and the N-terminal amino group of the proximal ubiquitin, Lys 63 is the closest to the C-terminal tail of the distal ubiquitin in our structure (Figure 1). This proximity of Lys 63 of the proximal ubiquitin to the C-terminal tail of the distal ubiquitin probably facilitates the specific recognition of Lys 63-linked polyubiquitin chains by RAP80-UIM1-UIM2. The next closest residue is the N-terminal methionine residue, whose amino group is $10 \AA$ away from the $\varepsilon$-amino group of Lys 63 . This proximity and the flexibility of the ubiquitin C-terminal tail could raise a question of how Lys 63-linked and linear ubiquitin chains can be discriminated. Further, crystal structures of $\mathrm{K} 63-\mathrm{Ub}_{2}$ and linear $\mathrm{Ub}_{2}$ suggested that two ubiquitin moieties could be oriented in almost the same geometry between both chains in isolation (Komander et al, 2009). However, our structure and a recent structure of a complex with linear $\mathrm{Ub}_{2}$ show that this $10 \AA$ Aifference is likely sufficient to discriminate both chains. The UBAN (ubiquitin binding in ABIN and NEMO) motif has been recently shown to selectively bind to linear $\mathrm{Ub}_{2}$ but not to $\mathrm{K} 63-\mathrm{Ub}_{2}$ (Rahighi et al, 2009). In the crystal structure of the NEMO UBAN domain in complex with linear $\mathrm{Ub}_{2}$, the peptide linkage between Gly 76 of the distal ubiquitin and Met 1 of the proximal ubiquitin is not directly recognized, although the proximal and distal ubiquitin moieties are simultaneously bound to the UBAN domain and specifically recognized in a distinct manner (Rahighi et al, 2009). The docking analysis using $\mathrm{K} 63-\mathrm{Ub}_{2}$ based on this complex structure showed that a $2 \AA$ shift of the terminal amino group of the proximal ubiquitin in the $\mathrm{K} 63-\mathrm{Ub}_{2}$ binding mode affects the interactions with critical residues, which probably weakens the binding of the 
A
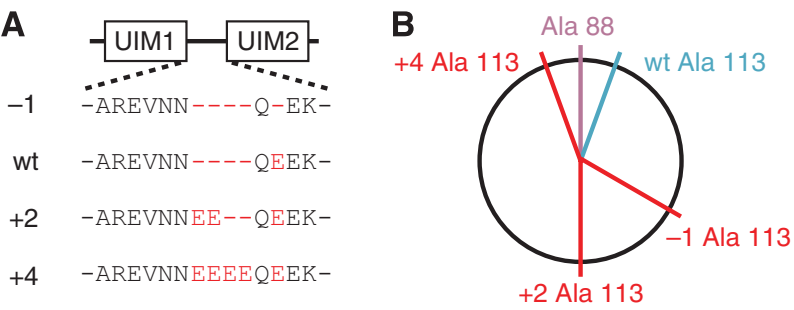

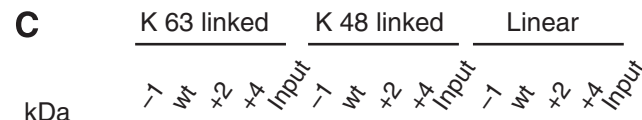

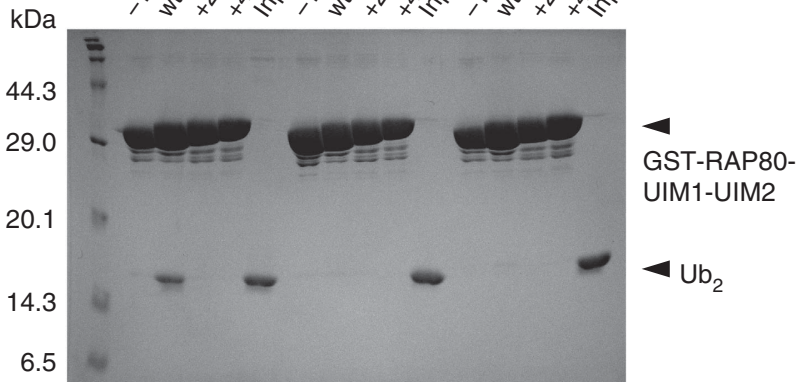

Figure 4 The length of the inter-UIM region of RAP80-UIM1-UIM2 is critical for its ability to bind $\mathrm{K} 63-\mathrm{Ub}_{2}$. (A) Amino-acid sequences of the inter-UIM region of the RAP80 mutants used for the GST pulldown assay. (B) Schematic view of residue positions in RAP80UIM1-UIM2. Ala 88 and Ala 113, the centre of the ubiquitin-binding surfaces of the RAP80 UIM1 and UIM2, are coloured pink and cyan, respectively. The putative positions of Ala 113 in the $-1,+2$, and +4 mutants of RAP80 are coloured red. (C) Pull-down assays using GST-fused RAP80-UIM1-UIM2 mutants and K63-, K48-, or linear $\mathrm{Ub}_{2}$

proximal ubiquitin to the UBAN motif (Rahighi et al, 2009). Although the orientation of the ubiquitin moieties in linear $\mathrm{Ub}_{2}$ bound to UBAN is totally different from that in $\mathrm{K} 63-\mathrm{Ub}_{2}$ bound to RAP80-UIM1-UIM2, a $10 \AA$ shift of the isopeptide/ peptide linkage estimated from our structure may be energetically disadvantageous for the $\mathrm{C}$-terminal tail conformation in the distal ubiquitin and affect the co-operative binding of RAP80-UIM1-UIM2 with di-ubiquitin. In this context, Leu 73 in the distal ubiquitin, which contacts with the interhelical region of RAP80-UIM1-UIM2 through van-der-Waals interaction, seems to partially restrict the C-terminal tail flexibility of the distal ubiquitin and may contribute to the linkage specificity.

\section{Tandem UIMs}

Several proteins that contain tandem UIMs have been previously identified (Figure 5A) (Hofmann and Falquet, 2001). For example, Epsin1 contains triple tandem UIMs, UIM1 (residues 183-198), UIM2 (residues 208-223), and UIM3 (residues 233-248) and the length of the two inter-UIM regions is the same as that of RAP80. Epsin1 interacts with the polyubiquitinated EGF receptor and promotes endocytosis of the polyubiquitinated EGF receptor in vivo (Sugiyama et al, 2005; Hawryluk et al, 2006; Kazazic et al, 2009). The tandem UIMs of Epsin1 have been reported to interact with both Lys 48-linked and Lys 63-linked polyubiquitin chains but to bind mono-ubiquitin very weakly (Sugiyama et al, 2005; Hawryluk et al, 2006). However, given that our work suggests that the length of the inter UIM region determines the Lys 63-linkage specificity of RAP80, we initially expected both pairs of tandem UIMs (i.e. UIM1-UIM2 and UIM2UIM3) of Epsin1 to selectively bind $\mathrm{K} 63-\mathrm{Ub}_{2}$. To analyze the specificity of ubiquitin binding by Epsin1 UIMs in the context of our structure, we carried out GST pull-down assays using UIM1-UIM2 (residues 183-224) and UIM2-UIM3 (residues 208-249) of Epsin1 fused to GST. As shown in Figure 5B, the UIM2-UIM3 of Epsin1 specifically binds K63$\mathrm{Ub}_{2}$, but the UIM1-UIM2 does not bind any of the ubiquitin linkages tested.

As mentioned above, the inter-UIM region of RAP80-UIM1UIM2 consists of an $\alpha$-helix, which rigidly determines the distance between and relative orientation of the ubiquitin binding sites of UIM1 and UIM2 to ensure specific $\mathrm{K} 63-\mathrm{Ub}_{2}$ binding (Figure 1C). The inter-UIM region between UIM2 and UIM3 of Epsin1 contains no amino-acid residues that distort the $\alpha$-helical structure, such as Tyr, Thr, Gly, Ser, and Pro (Figure 5A) (Levitt, 1978). However, the inter-UIM region between the UIM1 and UIM2 of Epsin 1 contains one serine, one glycine, and three proline residues (Figure 5A), which probably prevent this inter-UIM region from forming an $\alpha$ helical structure. These results suggest that in addition to the length of the inter-UIM region, the $\alpha$-helical structure of the inter-UIM region between tandem UIMs is essential for selective binding of Lys 63-linked polyubiquitin chains. Intriguingly, a splice variant of Epsin1 lacks a 25-residue segment, including UIM2 and lacks Pro, Ser and Gly residues in the inter-UIM region between UIM1 and UIM2 (Supplementary Figure S3) (Sugiyama et al, 2005). The length of the inter-UIM region between UIM1 and UIM3 of this splicing variant is the same as that of RAP80-UIM1-UIM2. In fact, this splicing variant, UIM1-UIM3, is functional in vivo (Sugiyama et al, 2005) and selectively binds with $\mathrm{K} 63-\mathrm{Ub}_{2}$ as shown in Figure 5.

\section{Comparison with other proteins that recognize Lys 63-linked ubiquitin chains}

The structural basis of the recognition of specific ubiquitin linkages by DUBs has been studied extensively (reviewed in Reyes-Turcu and Wilkinson, 2009) and crystal structures of the catalytic domains of the two Lys 63-linkage-specific DUBs, AMSH-LP and CYLD, have been reported (Komander et al, 2008; Sato et al, 2008). In the structure of the catalytic DUB domain of AMSH-LP bound to K63- $\mathrm{Ub}_{2}$, the AMSH-LP DUB domain interacts with both proximal and distal ubiquitin moieties and the roles of these two interactions are distinct in the context of the Lys 63-linkage-specific de-ubiquitination (Sato et al, 2008). The interaction with the distal ubiquitin is extensive and primarily contributes to the affinity for di-ubiquitin, whereas the interaction with the proximal ubiquitin is limited to regions near the isopeptidelinked lysine residue and facilitates the correct orientation and accurate positioning of the proximal ubiquitin for efficient linkage-specific de-ubiquitination. This mechanism was supported by kinetic analyses of the DUB reaction by AMSHLP (Sato et al, 2008). A similar mechanism has been suggested for CYLD, based on its crystal structure and mutagenesis experiments (Komander et al, 2008). Finally, the DUB OTU1 seems to adopt a similar strategy for Lys 48-linkagespecific de-ubiquitination (Wang et al, 2009).

The structural basis of selective ubiquitin-chain binding by UIMs was previously not understood in any context. Our work shows that RAP80-UIM1-UIM2 interacts with the distal 


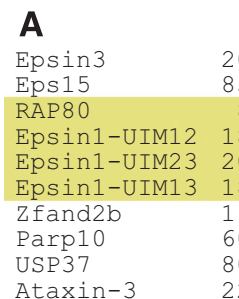

Ataxin-3

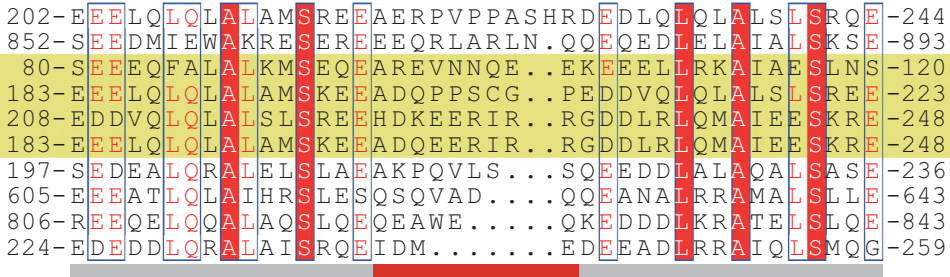

B

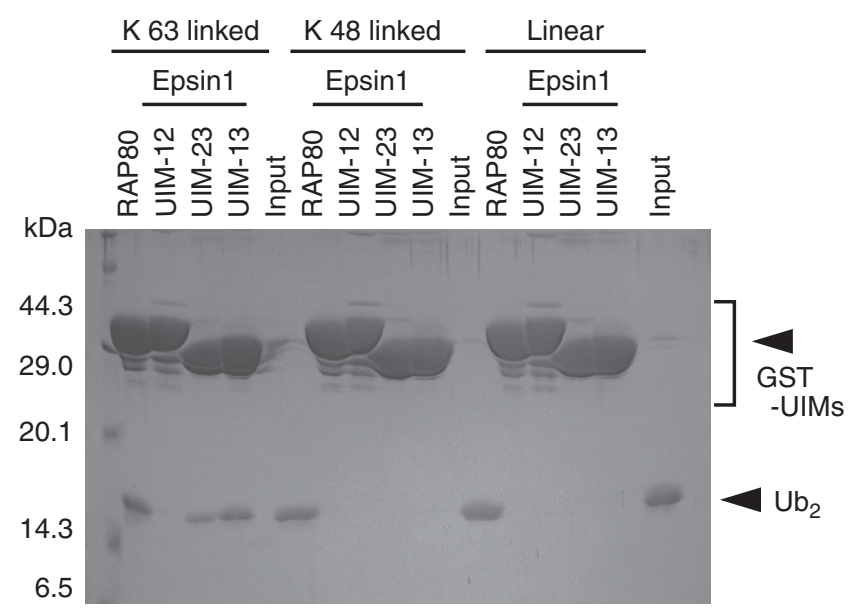

Figure 5 The tandem UIMs of Epsin1 specifically binds to K63-Ub 2 . The labels UIM-12, UIM-23, and UIM-13 represent UIM1-UIM2, UIM2UIM3 and UIM1-UIM3 of Epsin1, respectively. (A) Amino-acid sequence alignment of the tandem UIMs identified to date in the mouse genome. The symbols and colouring are the same as in Figure 3A. RAP80 and Epsin1 are highlighted by yellow background. (B) Pull-down assays using GST-fused RAP80-UIM1-UIM2 and Epsin1 to bind K63-, K48-, or linear $\mathrm{Ub}_{2}$.

and proximal ubiquitin moieties in essentially the same manner. Consistent with this, we find that the order of UIM1 and UIM2 in RAP80 is not critical for linkage-specific recognition. Importantly, neither UIM1 nor UIM2 directly interacts with the isopeptide-linked Lys 63. Instead, linkage specificity is determined by the inter-UIM distance and the relative orientation of UIMs matching that of the proximal and distal ubiquitin moieties. The inter-UIM region essentially acts as a molecular ruler by forming a $12 \AA$-long $\alpha$-helix to accurately measure the inter-ubiquitin distance and orientation, and specifically recognizes Lys 63-linked polyubiquitin chains. Furthermore, we have shown that this mechanism is employed by UIM2-UIM3 of Epsin1, whose inter-UIM region probably forms an $\alpha$-helical structure that is very similar to that of RAP80-UIM1-UIM2.

Here, we report the structural basis of Lys 63-linkagespecific recognition by tandem UIMs. However, it has been reported that a single UIM can determine linkage specificity in some cases. For instance, the UIM of the Lys 63-linkagespecific DUBs DUBA and ataxin3 is required for selective cleavage of Lys 63-linked polyubiquitin chains (Kayagaki et al, 2007; Winborn et al, 2008). Further, the UIM of Met4 selectively binds Lys 48-linked polyubiquitin chains (Flick et al, 2006). Structural analyses of these UIM-containing proteins may elucidate new mechanisms for the determination of linkage selectivity by UIMs.

\section{Materials and methods}

\section{Preparation of the RAP80-UIM1-UIM2-K63-Ub ${ }_{2}$ complex}

The genes encoding RAP80 and Epsin1 were PCR amplified from a mouse cDNA library. The amplified PCR products were cloned into the pGEX-6P-1 expression vector (GE Healthcare) with BamHI and $X$ hoI sites to produce an N-terminal GST fusion protein and were confirmed by DNA sequencing. Escherichia coli strain Rosetta (DE3) cells (Invitrogen) were transformed with the expression vector, and were cultured in LB containing $100 \mathrm{mg} / \mathrm{l}$ ampicillin at $37^{\circ} \mathrm{C}$. Recombinant protein expression was induced by the addition of $0.3 \mathrm{mM}$ isopropyl- $\beta$-D-thiogalactopyranoside at an $\mathrm{A}_{600}$ of 0.5 , and then the E. coli cells were incubated overnight at $20^{\circ} \mathrm{C}$. The cells were collected by centrifugation at $8000 \mathrm{~g}$ for $15 \mathrm{~min}$ and were disrupted by sonication in phosphate buffered saline (PBS), containing $1 \mathrm{mM}$ dithiothreitol (DTT), $1 \mathrm{mM}$ phenylmethylsulfonyl fluoride (PMSF), and $0.5 \%$ Triton X-100. The lysates were centrifuged at $30000 \mathrm{~g}$ for $60 \mathrm{~min}$ and the supernatants were then loaded onto a Glutathione Sepharose FF column (GE Healthcare), which had been pre-equilibrated with PBS containing $1 \mathrm{mM}$ DTT and $0.5 \%$ Triton X-100. The column was washed with PBS containing $1 \mathrm{mM}$ DTT and $0.5 \%$ Triton X-100 and then with PBS containing $1 \mathrm{mM}$ DTT. GST fusion proteins were eluted with $50 \mathrm{mM}$ Tris-HCl buffer (pH 8.0), containing $200 \mathrm{mM} \mathrm{NaCl}, 1 \mathrm{mM}$ DTT, and $15 \mathrm{mM}$ reduced glutathione. The GST tags were cleaved by PreScission protease (GE Healthcare), and the samples were dialyzed against $50 \mathrm{mM}$ Tris- $\mathrm{HCl}$ buffer $(\mathrm{pH}$ 8.0), containing $40 \mathrm{mM} \mathrm{NaCl}$ and $1 \mathrm{mM}$ DTT. The proteins were loaded onto a ResourceQ anion exchange column (GE Healthcare) and were eluted using a linear gradient of $0-1 \mathrm{M} \mathrm{NaCl}$. The cleaved GST tags were then removed by chromatography on a Glutathione Sepharose FF column. To prepare the RAP80-UIM1-UIM2-K63-Ub 2 complex, a two-fold molar excess of RAP80-UIM1-UIM2 was incubated at $4{ }^{\circ} \mathrm{C}$ overnight with $\mathrm{K} 63-\mathrm{Ub}_{2}$ that was prepared as described previously (Sato et al, 2008). The RAP80-UIM1-UIM2-K63-Ub 2 complex was loaded onto a HiLoad Superdex 75 size-exclusion column (GE Healthcare) pre-equilibrated with $10 \mathrm{mM}$ Tris-HCl buffer ( $\mathrm{pH} 7.2$ ), $50 \mathrm{mM} \mathrm{NaCl}$, and $5 \mathrm{mM} \beta$-mercaptoethanol to remove the unbound RAP80-UIM1-UIM2.

\section{Crystallization and data collection}

The purified RAP80-UIM1-UIM2-K63-Ub 2 complex was concentrated to $10.5 \mathrm{~g} / \mathrm{l}$, by using an Amicon Ultra-15 3000 MWCO filter (Millipore) following the manufacturer's instructions. The initial 
Table I Data collection and refinement statistics

\begin{tabular}{|c|c|}
\hline \multicolumn{2}{|l|}{ Data collection } \\
\hline $\mathrm{X}$-ray source & SPring8 BL41XU \\
\hline Wavelength $(\AA)$ & 1.0000 \\
\hline Space group & $P 2_{1} 2_{1} 2_{1}$ \\
\hline Unit-cell parameter & $\begin{array}{c}a=65.2 \AA ; b=74.8 \AA ; \\
c=83.0 \AA\end{array}$ \\
\hline Resolution $(\AA)$ & $50.0-2.20(2.23-2.20)$ \\
\hline Unique reflections & 21280 \\
\hline Total reflections & 762438 \\
\hline Completeness (\%) & $94.7(88.2)$ \\
\hline$I / \sigma(I)$ & $10.7(2.6)$ \\
\hline$R_{\text {sym }}$ & $0.098(0.340)$ \\
\hline \multicolumn{2}{|l|}{ Refinement } \\
\hline Number of atoms: protein, water & 3091,156 \\
\hline r.m.s.d. bond length $(\AA)$ & 0.006 \\
\hline r.m.s.d. bond angle (deg) & 1.28 \\
\hline Average $B$ factors: protein, water & $30.1,32.4$ \\
\hline Residues in core region (\%) & 95.9 \\
\hline $\begin{array}{l}\text { Residues in additionally allowed } \\
\text { region (\%) }\end{array}$ & 4.1 \\
\hline $\begin{array}{l}\text { Residues in generously allowed re- } \\
\text { gion }(\%)\end{array}$ & 0.0 \\
\hline Residues in disallowed region (\%) & 0.0 \\
\hline$R_{\text {work }}, R_{\text {free }}$ & $0.216,0.268$ \\
\hline
\end{tabular}

The numbers in parentheses are for the highest resolution shell.

$R_{\text {sym }}=\Sigma\left|I_{\text {avg }}-I_{\mathrm{i}}\right| / \Sigma I_{\mathrm{i}}$.

$R_{\text {cullis }}=\Sigma|| \mathbf{F}_{\mathrm{PH}}-\mathbf{F}_{\mathrm{P}}|-| \mathbf{F}_{\mathrm{H}(\text { calc })}|| / \Sigma\left|\mathbf{F}_{\mathrm{PH}}\right|$.

$R_{\mathrm{work}}=\Sigma\left|F_{\mathrm{o}}-F_{\mathrm{c}}\right| / \Sigma F_{\mathrm{o}}$ for reflections of working set.

$R_{\text {free }}=\Sigma\left|F_{\mathrm{o}}-F_{\mathrm{c}}\right| / \Sigma F_{\mathrm{o}}$ for reflections of test set (5\% of total unique reflections).

crystallization screening was carried out using the sitting-drop vapour diffusion method at $20^{\circ} \mathrm{C}$ using a Mosquito ${ }^{\circledR}$ liquid-handling robot (TTP Lab Tech). We tested about 500 conditions, using crystallization reagent kits supplied by Hampton Research, and initial hits were further optimized. The best crystals of the RAP80UIM1-UIM2-K63-Ub $b_{2}$ complex were obtained at $20^{\circ} \mathrm{C}$ with the sitting-drop vapour diffusion method by mixing $1 \mu \mathrm{l}$ of protein solution with an equal amount of precipitant solution containing $90 \mathrm{mM}$ Tris- $\mathrm{HCl}$ buffer (pH 8.5), $180 \mathrm{mM}$ sodium acetate, $29 \%$ PEG4000, and $0.5 \%$ polyvinylpyrrolidone $\mathrm{K} 15$, and equilibrating against $500 \mu \mathrm{l}$ of reservoir solution containing $100 \mathrm{mM}$ Tris- $\mathrm{HCl}$ buffer ( $\mathrm{pH}$ 8.5), $200 \mathrm{mM}$ sodium acetate, and $32 \%$ PEG4000. The crystal of the RAP80-UIM1-UIM2-K63-Ub 2 complex, which was used for the final structure refinement, belongs to the space group $P 2_{1} 2_{1} 2_{1}$, with unit cell parameters, $a=65.2 \AA, b=74.8 \AA, c=83.0 \AA$. Diffraction data for the RAP80-UIM1-UIM2-K63-Ub 2 complex crystal were collected at the beamline BL41XU in SPring-8 (Hyogo, Japan), and were processed using the program HKL2000 (Otwinowski and Minor, 1997) and the CCP4 program suite (Collaborative Computational Project, 1994).

\section{References}

Brunger AT, Adams PD, Clore GM, DeLano WL, Gros P, Grosse-Kunstleve RW, Jiang JS, Kuszewski J, Nilges M, Pannu NS, Read RJ, Rice LM, Simonson T, Warren GL (1998) Crystallography \& NMR system: a new software suite for macromolecular structure determination. Acta Crystallogr D 54: 905-921

Brzovic PS, Keeffe JR, Nishikawa H, Miyamoto K, Fox III D, Fukuda M, Ohta T, Klevit R (2003) Binding and recognition in the assembly of an active BRCA1/BARD1 ubiquitin-ligase complex. Proc Natl Acad Sci USA 100: 5646-5651

Christensen DE, Brzovic PS, Klevit RE (2007) E2-BRCA1 RING interactions dictate synthesis of mono- or specific polyubiquitin chain linkages. Nat Struct Mol Biol 14: 941-948

Collaborative Computational Project N (1994) The CCP4 Suite: Programs for Protein Crystallography. Acta Crystallogr D 50: $760-763$

\section{Structural determination and refinement}

The structure of the RAP80-UIM1-UIM2-K63-Ub 2 complex was determined using molecular replacement using the program Molrep (Vagin and Teplyakov, 1997). A search model was generated from the structure of the Hrs-UIM bound to two ubiquitin molecules (PDB code: $2 \mathrm{D} 3 \mathrm{G}$ ) by removing all water molecules and removing the ubiquitin molecule that was assigned as Chain B. An automated rotation and translation search found a clear solution with an $R$-factor of $46.2 \%$ and a correlation coefficient of 0.554 in the resolution range of $50-2.7 \AA$. Four UIM-ubiquitin complexes were found in the asymmetric unit. The model was built and refined up to $2.2 \AA$ resolution using the programs COOT (Emsley and Cowtan, 2004) and CNS (Brunger et al, 1998). Data collection, phasing, and refinement statistics are shown in Table I. All molecular graphics were prepared using the program PyMOL (DeLano Scientific; http://www.pymol.org).

For GST pull-down assays, GST-fused UIMs of RAP80 and Epsin1 were overproduced in E. coli strain Rosetta ${ }^{\mathrm{TM}}$ (DE3) (Invitrogen), and purified by Glutathione Sepharose FF and Resource Q anion exchange columns (GE Healthcare). The mutations were generated by PCR. The GST-fused wild-type or mutant for RAP80 or Epsin1 UIMs was immobilized on Glutathione Sepharose FF beads, and then incubated with $\mathrm{K} 63-$-, $\mathrm{K} 48-$, or linear $\mathrm{Ub}_{2}$ for $15 \mathrm{~min}$ at $4^{\circ} \mathrm{C}$ on ice in phosphate buffered saline containing $0.1 \%$ Triton X-100. The beads were extensively washed with the same buffer thrice. The diubiquitin molecules bound to the beads were released by boiling in SDS loading buffer, analyzed by SDS-PAGE, and stained using Coomassie brilliant blue stain.

\section{Supplementary data}

Supplementary data are available at The EMBO Journal Online (http://www.embojournal.org).

\section{Acknowledgements}

We thank C Toyoshima for supporting this research; S Kaiser for critical reading and improvement of this paper. We are grateful to $\mathrm{K}$ Iwai for providing us with reagents for the overexpression of $\mathrm{E} 1$, E2-25K, and linear $\mathrm{Ub}_{2}$; $\mathrm{M}$ Komada for providing us with the overexpression vectors of ubiquitin mutants for $\mathrm{K} 63-\mathrm{Ub}_{2}$ and $\mathrm{K} 48$ $\mathrm{Ub}_{2}$ synthesis. We thank the beamline staffs at BL-5A of Photon Factory (Tsukuba, Japan) and BL41XU of SPring8 (Hyogo, Japan) for technical help during data collection. This study was supported by grants from MEXT to SF, A Ya, and HM. YS and MY are supported by JSPS research fellowships for young scientists. The coordinates and structure factors of RAP80-UIM1-UIM2 in complex with K63$\mathrm{Ub}_{2}$ have been deposited in the Protein Data Bank with accession codes 3A1Q.

\section{Conflict of interest}

The authors declare that they have no conflict of interest.

Doil C, Mailand N, Bekker-Jensen S, Menard P, Larsen DH, Pepperkok R, Ellenberg J, Panier S, Durocher D, Bartek J, Lukas J, Lukas C (2009) RNF168 binds and amplifies ubiquitin conjugates on damaged chromosomes to allow accumulation of repair proteins. Cell 136: 435-446

Emsley P, Cowtan K (2004) Coot: model-building tools for molecular graphics. Acta Crystallogr D 60: 2126-2132

Fisher RD, Wang B, Alam SL, Higginson DS, Robinson H, Sundquist WI, Hill CP (2003) Structure and ubiquitin binding of the ubiquitin-interacting motif. J Biol Chem 278: 28976-28984

Flick K, Raasi S, Zhang H, Yen JL, Kaiser P (2006) A ubiquitininteracting motif protects polyubiquitinated Met4 from degradation by the $26 \mathrm{~S}$ proteasome. Nat Cell Biol 8: $509-515$

Glickman MH, Ciechanover A (2002) The ubiquitin-proteasome proteolytic pathway: destruction for the sake of construction. Physiol Rev 82: 373-428 
Hawryluk MJ, Keyel PA, Mishra SK, Watkins SC, Heuser JE, Traub LM (2006) Epsin 1 is a polyubiquitin-selective clathrin-associated sorting protein. Traffic 7: 262-281

Hicke L (2001) Protein regulation by monoubiquitin. Nat Rev Mol Cell Biol 2: 195-201

Hirano S, Kawasaki M, Ura H, Kato R, Raiborg C, Stenmark H, Wakatsuki S (2006) Double-sided ubiquitin binding of Hrs-UIM in endosomal protein sorting. Nat Struct Mol Biol 13: 272-277

Hofmann K (2009) Ubiquitin-binding domains and their role in the DNA damage response. DNA Repair (Amst) 8: 544-556

Hofmann K, Falquet L (2001) A ubiquitin-interacting motif conserved in components of the proteasomal and lysosomal protein degradation systems. Trends Biochem Sci 26: 347-350

Hurley JH, Lee S, Prag G (2006) Ubiquitin-binding domains. Biochem J 399: 361-372

Kayagaki N, Phung Q, Chan S, Chaudhari R, Quan C, O’Rourke KM, Eby M, Pietras E, Cheng G, Bazan JF, Zhang Z, Arnott D, Dixit VM (2007) DUBA: a deubiquitinase that regulates type I interferon production. Science 318: 1628-1632

Kazazic M, Bertelsen V, Pedersen KW, Vuong TT, Grandal MV, Rodland MS, Traub LM, Stang E, Madshus IH (2009) Epsin 1 is involved in recruitment of ubiquitinated EGF receptors into clathrin-coated pits. Traffic 10: 235-245

Kim $\mathrm{H}$, Chen J, Yu X (2007) Ubiquitin-binding protein RAP80 mediates BRCA1-dependent DNA damage response. Science 316: $1202-1205$

Kinner A, Wu W, Staudt C, Iliakis G (2008) Gamma-H2AX in recognition and signaling of DNA double-strand breaks in the context of chromatin. Nucleic Acids Res 36: 5678-5694

Kirisako T, Kamei K, Murata S, Kato M, Fukumoto H, Kanie M, Sano S, Tokunaga F, Tanaka K, Iwai K (2006) A ubiquitin ligase complex assembles linear polyubiquitin chains. EMBO $J \mathbf{2 5}$ : 4877-4887

Komander D, Lord CJ, Scheel H, Swift S, Hofmann K, Ashworth A, Barford D (2008) The structure of the CYLD USP domain explains its specificity for Lys63-linked polyubiquitin and reveals a B Box module. Mol Cell 29: 451-464

Komander D, Reyes-Turcu F, Licchesi JD, Odenwaelder P, Wilkinson KD, Barford D (2009) Molecular discrimination of structurally equivalent Lys 63-linked and linear polyubiquitin chains. EMBO Rep 10: 466-473

Levitt M (1978) Conformational preferences of amino acids in globular proteins. Biochemistry 17: 4277-4285

Meierhofer D, Wang X, Huang L, Kaiser P (2008) Quantitative analysis of global ubiquitination in HeLa cells by mass spectrometry. J Proteome Res 7: 4566-4576

Miranda M, Sorkin A (2007) Regulation of receptors and transporters by ubiquitination: new insights into surprisingly similar mechanisms. Mol Interv 7: 157-167

Otwinowski Z, Minor W (1997) Processing of X-ray diffraction data collected in oscillation mode. Methods Enzymol 276: 307-326

Panier S, Durocher D (2009) Regulatory ubiquitylation in response to DNA double-strand breaks. DNA Repair (Amst) 8: 436-443

Rahighi S, Ikeda F, Kawasaki M, Akutsu M, Suzuki N, Kato R, Kensche T, Uejima T, Bloor S, Komander D, Randow F, Wakatsuki S, Dikic I (2009) Specific recognition of linear ubiquitin chains by NEMO is important for NF-kappaB activation. Cell 136: 1098-1109

Reyes-Turcu FE, Wilkinson KD (2009) Polyubiquitin binding and disassembly by deubiquitinating enzymes. Chem Rev 109: 1495-1508

Sato Y, Yoshikawa A, Yamagata A, Mimura H, Yamashita M, Ookata K, Nureki O, Iwai K, Komada M, Fukai S (2008) Structural basis for specific cleavage of Lys 63-linked polyubiquitin chains. Nature 455: $358-362$

Shao G, Lilli DR, Patterson-Fortin J, Coleman KA, Morrissey DE, Greenberg RA (2009) The Rap80-BRCC36 de-ubiquitinating enzyme complex antagonizes RNF8-Ubc13-dependent ubiquitination events at DNA double strand breaks. Proc Natl Acad Sci USA 106: $3166-3171$

Sims JJ, Cohen RE (2009) Linkage-specific avidity defines the lysine 63-linked polyubiquitin-binding preference of rap80. Mol Cell 33: $775-783$

Sobhian B, Shao G, Lilli DR, Culhane AC, Moreau LA, Xia B, Livingston DM, Greenberg RA (2007) RAP80 targets BRCA1 to specific ubiquitin structures at DNA damage sites. Science 316: 1198-1202

Stewart GS, Panier S, Townsend K, Al-Hakim AK, Kolas NK, Miller ES, Nakada S, Ylanko J, Olivarius S, Mendez M, Oldreive C, Wildenhain J, Tagliaferro A, Pelletier L, Taubenheim N, Durandy A, Byrd PJ, Stankovic T, Taylor AM, Durocher D (2009) The RIDDLE syndrome protein mediates a ubiquitindependent signaling cascade at sites of DNA damage. Cell 136: $420-434$

Sugiyama S, Kishida S, Chayama K, Koyama S, Kikuchi A (2005) Ubiquitin-interacting motifs of Epsin are involved in the regulation of insulin-dependent endocytosis. J Biochem 137: 355-364

Swanson KA, Kang RS, Stamenova SD, Hicke L, Radhakrishnan I (2003) Solution structure of Vps27 UIM-ubiquitin complex important for endosomal sorting and receptor downregulation. EMBO J 22: 4597-4606

Vagin A, Teplyakov A (1997) MOLREP: an automated program for molecular replacement. J Appl Cryst 30: 1022-1025

Verstrepen L, Bekaert T, Chau TL, Tavernier J, Chariot A, Beyaert R (2008) TLR-4, IL-1R and TNF-R signaling to NF-kappaB: variations on a common theme. Cell Mol Life Sci 65: 2964-2978

Wang B, Elledge SJ (2007) Ubc13/Rnf8 ubiquitin ligases control foci formation of the Rap80/Abraxas/Brca1/Brcc36 complex in response to DNA damage. Proc Natl Acad Sci USA 104: 20759-20763

Wang B, Matsuoka S, Ballif BA, Zhang D, Smogorzewska A, Gygi SP, Elledge SJ (2007) Abraxas and RAP80 form a BRCA1 protein complex required for the DNA damage response. Science 316: 1194-1198

Wang Q, Young P, Walters KJ (2005) Structure of S5a bound to monoubiquitin provides a model for polyubiquitin recognition. J Mol Biol 348: 727-739

Wang T, Yin L, Cooper EM, Lai MY, Dickey S, Pickart CM, Fushman D, Wilkinson KD, Cohen RE, Wolberger C (2009) Evidence for bidentate substrate binding as the basis for the K48 linkage specificity of otubain 1. J Mol Biol 386: 1011-1023

Wickliffe K, Williamson A, Jin L, Rape M (2009) The multiple layers of ubiquitin-dependent cell cycle control. Chem Rev 109: 1537-1548, doi:10.1021/cr800414e

Winborn BJ, Travis SM, Todi SV, Scaglione KM, Xu P, Williams AJ, Cohen RE, Peng J, Paulson HL (2008) The deubiquitinating enzyme ataxin-3, a polyglutamine disease protein, edits Lys63 linkages in mixed linkage ubiquitin chains. J Biol Chem 283: 26436-26443

Xu P, Duong DM, Seyfried NT, Cheng D, Xie Y, Robert J, Rush J, Hochstrasser M, Finley D, Peng J (2009) Quantitative proteomics reveals the function of unconventional ubiquitin chains in proteasomal degradation. Cell 137: 133-145

Yan J, Jetten AM (2008) RAP80 and RNF8, key players in the recruitment of repair proteins to DNA damage sites. Cancer Lett 271: 179-190

Yan J, Kim YS, Yang XP, Li LP, Liao G, Xia F, Jetten AM (2007) The ubiquitin-interacting motif containing protein RAP80 interacts with BRCA1 and functions in DNA damage repair response. Cancer Res 67: 6647-6656

Young P, Deveraux Q, Beal RE, Pickart CM, Rechsteiner M (1998) Characterization of two polyubiquitin binding sites in the 26S protease subunit 5a. J Biol Chem 273: 5461-5467 